\section{THE RESPIRATOR-INHALER.}

By WILLIAM ROBERTS, M.D., F.R.C.P., Physician to the Manchester Infirmary; Professor of Clinical Medicine to the Owens College ; etc.

THE respirator-inhaler is designed to facilitate the use of topical applications to the respiratory tract, and the administration of remedies by absorption through the pulmonary surfaces. It consists of a shallow tin box, of the size and shape of the ordinary respirator. The top and bottom are perforated with holes like the lid of a pepper-box. The box is fitted with some porous material (tow, oakum, or very open flannel), and the top lifts off with a hinge, to permit the renewal of the porous material and the addition of whatever drug the prescriber may wish the patient to inhale. Five, ten, or more drops of some volatile drug are dropped on the porous material, and the box is closed and fastened on, like a respirator, by means of elastic bands drawn round the ears.

The instrument, thus charged, is worn for fifteen, thirty, or sixty minutes several times a day ; and additional quantities of the drug are introduced into the porous material from time to time as may be deemed necessary. The porous material also requires renewing occasionally.

The object aimed at is to obtain the effects of small quantities of the drug, slowly and continuously applied, either for the purpose of a topical application to some part of the respiratory mucous membrane, or for direct absorption of the drug into the blood. Of course, only volatile remedies can be used in this way. As the patient continues to breathe to and fro through the drug-charged porous material, the contents of the box become warmed, and the volatile ingredient is vaporised and passes with the breath into the air-passages, and comes into contact with every part of the breathing surfaces.

The following are the substances I have hitherto used to charge the respirator-inhaler, according to the effects it was desired to obtain : Turpentine, oleum pini sylvestris, spirit of camphor, the oils of sandal wood and eucalyptus, compound tincture of benzoin, creasote, carbolic acid, iodine, and chloroform.

My experience is, as yet, too limited to enable me to formulate conclusions as to the probable value of the instrument ; but I will relate a few examples in illustration of the way in which $I$ have used it.

I. An apyrexial patient had a patch of congested lung under the right shoulder-blade, from which he continued to spit blood, in spite of sundry remedies, for some weeks. He was directed to inhale six drops of oil of turpentine from the respiratory inhaler for an hour three times a day. This was almost immediately followed by the cessation of the hæmorrhage. When the dose was increased to ten drops, the patient complained of a transient feeling resembling intoxication. In a second case of hæmoptysis, of tuberculous character, cessation of the hæmorrhage almost immediately followed the inhalation of turpentine in a similar manner.

2. A phthisical patient with cavities in both lungs, from which he expectorated large quantities of pus, inhaled four drops of creasote for an hour three times a day, with the apparent effect of reducing the quantity of pus to less than one-half.

3. Another phthisical patient inhaled, in a similar manner, six drops of the liquor iodi (P. B.), with great apparent benefit.

4. A patient with advanced laryngeal phthisis, under the care of my former pupil Mr. Hunt, appeared to derive relief from the inhalation of fifteen drops of a mixture of equal parts of chloroform and oil. The pain in swallowing and the tickling cough were markedly alleviated by the application.

5. In several other cases of advanced phthisis, with profuse purulent expectoration, I have failed to observe any good effects from the inhalation of carbolic acid and of iodine by means of the respirator-inhaler.

6. In cases of bronchitis, I have seen the hest results follow the in halation of the compound tincture of benzoin and the oleum pini sylvestris. Coal-tar and wood-tar would appear likewise suitable for cases of chronic bronchitis.

In apportioning the dosage, I have sought to avoid provoking cough or catching of the breath, using only such small quantities of the drug as the patient could inhale without a feeling of irritation. It is otherwise manifestly impossible to obtain a prolonged contact of the remedy with the respiratory surfaces.

The advantages which this method of inhaling appears to have over the ordinary methods consist in its being less burdensome to the patient, and in the prolonged contact of the remedy with the affected surfaces. The instrument may likewise be worn for the purposes of an ordinary respirator, and it is sufficiently cheap to be within reach of the humblest patient.

The respirator-inhaler may be had from J. and W. Wood, surgical instrument makers, King Street, Manchester. The instrument is made either plain or mounted with velvet. The plain instrument is the more useful, as it can be washed and cleaned in hot water; the mounted instrument is chiefly for outdoor use, as its appearance is scarcely more conspicuous than that of an ordinary respirator. The price of the former is three shillings and of the latter five shillings and sixpence.

The Messrs. Wood have also constructed a modification of this instrument, with an upward prolongation, to cover the nose as well as the mouth, like a chloroform-inhaler. This modification is designed for the use of children, and for persons who find it inconvenient to breathe for a long time through the mouth.

\section{LARYNGOTOMY IN CHLOROFORM ASPHYXIA.}

\section{By J. T. CLOVER, F.R.C.S.}

THE deaths under chloroform, recently reported, induce me, without further hesitation, to report a case in which death seems to have been prevented by laryngotomy ; and to make some remarks as to the precise time when that operation ought to be performed.

A gentleman, aged 60 , suffering from a large epithelioma of the tongue, had this removed last October, by Mr. Marshall, by means of the galvanic écraseur. A mixture of chloroform and ether induced sleep in six minutes. The patient was then kept breathing with light stertor five minutes longer before the face-piece was removed. From this time, chloroform without ether was given by covering the nose with a cap, supplied with vapour as the face-piece had been. In spite of this, the patient recovered sufficiently, during the arrangement of the ecraseur, to become unsteady at the moment when the wire was heated by the galvanic current. I gave more chloroform-vapour by the mouth, also supplied from the same source as that which supplied the nose. In a minute or more, the patient was quiet and continued so, breathing steadily, for ten minutes. At this time, the patient moved his wrist, as if trying to get it away from my grasp. I slightly increased the strength of the chloroform, without, however, producing either cough or swallowing ; and when the wrist became quiet, I decreased the supply of chloroform. Soon after this, I observed a pause in the breathing, and removed the chloroform-tube from the mouth; but, as his pulse was good, and as it was very important he should not struggle at this stage of the operation, I continued the supply by the nosecap for a few seconds longer. The pause in the breathing was of short duration, but, as the succeeding respirations were feeble, I took off the nosecap. There were but two or three respirations during the next thirty seconds, and they were feeble, and accompanied by a sound in the throat. Finding that his pulse re mained steady and firm, and believing that one singlegasp of fresh air would restore him as much as was desirable, I desisted from moving the chest until I perceived a lessening of the strength of the pulse. I now pressed upon the chest and abdomen, the patient being already lying on his back. As the pressure was removed, there was a sound of air passing through the larynx; but this sound gradually changed to a faint hiccough and then ceased. The tongue had been secured by a ligature at the beginning of the operation, and had beendrawn forcibly forward, in order to apply the loop of the écraseur. We now tried the Silvester method, with a similar failure ; for although the air passed through the larynx, at first audibly, after a few movements the sound became a short hiccough, and then ceased. After persevering for perhaps two minutes, in endeavouring to produce artificial respiration, but in vain, $\mathrm{Mr}$. Marshall and I thought it prudent to have recourse to laryngotomy. The radial pulse could not be felt. After the laryngeal tube was inserted, the Silvester method answered admirably. Air immediately entered, and escaped freely through the tube; some hæmorrhage from the wound in the neck necessitating repeated cleansing away of frothy blood from the external aperture of the tube. At this juncture, it was evident that the artificial respiratory acts, produced by the persistence in the Silvester method, were now rendered serviceable in emptying and filling the lungs through the tube, instead of failing to do so, as before, through the glottis. This was the turning point in the condition of the patient. Very soon, however, perhaps in a minute or more, a gasp through the glottis occurred, followed by another in ten seconds. From this time, breathing went on without the mechanical assistance of the Silvester method; but both inspiration and expiration through the glottis were visibly supplemented by the free simultaneous entrance and escape of air through the laryngeal tube.

The operation was then continued, the patient in three minutes be- 